\title{
SCUBA sub-millimeter observations of gamma-ray bursts
}

\section{GRB 030329: the brightest sub-millimeter afterglow to date}

\author{
I. A. Smith ${ }^{1}$, R. P. J. Tilanus ${ }^{2}$, N. Tanvir ${ }^{3}$, R. A. M. J. Wijers ${ }^{4}$, P. Vreeswijk ${ }^{5}$, E. Rol ${ }^{6}$, and C. Kouveliotou ${ }^{7,8}$ \\ 1 Department of Physics and Astronomy, Rice University, 6100 South Main, MS-108, Houston, TX 77005-1892, USA \\ e-mail: iansmith@rice.edu \\ 2 Joint Astronomy Centre, 660 N. Aohoku Place, Hilo, HI 96720, USA \\ 3 Centre for Astrophysics Research, University of Hertfordshire, College Lane, Hatfield, Herts AL10 9AB, UK \\ 4 Astronomical Institute "Anton Pannekoek", University of Amsterdam and Center for High-Energy Astrophysics, \\ Kruislaan 403, 1098 SJ Amsterdam, The Netherlands \\ 5 European Southern Observatory, Alonso de Córdova 3107, Casilla 19001, Santiago 19, Chile \\ ${ }^{6}$ Department of Physics and Astronomy, University of Leicester, Leicester LE1 7RH, UK \\ 7 NASA Marshall Space Flight Center, SD-50, NSSTC, 320 Sparkman Drive, Huntsville, AL 35805, USA \\ ${ }^{8}$ Universities Space Research Association
}

Received 21 March 2005 / Accepted 11 May 2005

\begin{abstract}
We present all the Target of Opportunity (ToO) sub-millimeter observations of GRB 030329 taken by the Sub-millimetre Common-User Bolometer Array (SCUBA) on the James Clerk Maxwell Telescope (JCMT). This was by far the brightest sub-millimeter afterglow seen to date. The flux density at $850 \mu \mathrm{m}$ was approximately constant up to a break that took place $\sim 7$ days after the burst. This was consistent with being a jet break. The $850 \mu \mathrm{m}$ results agree with those at longer wavelengths that show a brighter flux $\sim 7$ days after the burst, right at the time of the break. No short-lived large-scale brightenings were detected in the sub-millimeter light curve. However, the $850 \mu \mathrm{m}$ light curve may have had a drop $\lesssim 16$ days after the burst. The peak of the afterglow emission was at $\sim 90 \mathrm{GHz}$ in the days before the break in the light curve. A simple modeling is consistent with the spectral indices remaining the same as the afterglow evolved, with the breaks in the spectrum moving to longer wavelengths at later times and the flux at the peak falling. No significant sub-millimeter emission was detected from the host galaxy.
\end{abstract}

Key words. gamma rays: bursts - submillimeter

\section{Introduction}

The discovery of localized transients in the error boxes of gamma-ray burst (GRB) sources has led to intense multiwavelength campaigns that have revolutionized our understanding of these sources. For reviews see Van Paradijs et al. (2000) and Mészáros (2002).

Sub-millimeter observations form a key element of the multiwavelength observations of the afterglow. They provide "clean" measures of the source intensity, unaffected by scintillation and extinction. We have therefore been performing Target of Opportunity (ToO) sub-millimeter observations of GRB counterparts using the Sub-millimetre Common-User Bolometer Array (SCUBA) on the James Clerk Maxwell Telescope (JCMT) on Mauna Kea, Hawaii.

Including the results shown here, SCUBA has so far performed ToO observations of 23 bursts (Smith et al. 1999, 2000, 2001, 2005; Bloom et al. 1998; Galama et al. 1999; Kulkarni et al. 1999; Frail et al. 2000, 2002, 2003; Berger et al. 2000, 2003a; Yost et al. 2002). A complete summary of the previous
SCUBA results and the motivations for performing submillimeter observations of the afterglows and host galaxies is given in Smith et al. (2005). In this paper, we present all the SCUBA ToO observations of GRB 030329.

\section{Multiwavelength observations of GRB 030329}

The long (>100 s) GRB 030329 was detected by HETE-2 and several spacecraft in the Interplanetary Network at 20030329.484 UT (Vanderspek et al. 2004). The intense ionizing flux from the burst produced a sudden disturbance to the Earth's ionosphere (Schnoor et al. 2003).

The redshift was determined to be 0.1685 (Greiner et al. 2003a), corresponding to a luminosity distance of $\sim 810 \mathrm{Mpc}$. This was one of the closest GRBs localized to date. Although its intrinsic luminosity was relatively low, the fact that it was nearby made it one of the brightest bursts and afterglows ever recorded.

GRB 030329 was an X-ray rich burst, and a distinct, bright, soft X-ray component may have been present during 
the burst. The X-ray afterglow seen by the Rossi X-Ray Timing Explorer and XMM-Newton was bright (Marshall \& Swank 2003; Marshall et al. 2003; Tiengo et al. 2003, 2004). However, the X-ray afterglow light curve was sparsely sampled. It might extrapolate back to match the soft X-ray tail seen during the burst. There appeared to be a break in the decay of the X-ray light curve $\sim 0.5$ days after the burst, and there may have been a flattening of the X-ray light curve $\sim 40$ days after the burst.

Optical observations simultaneous with the burst did not detect the source, placing a limit of $V \sim 5.5$ on the reverse shock emission (Torii et al. 2003). However, a bright optical afterglow was found, with $R \sim 12.4$ at $67 \mathrm{~min}$ after the burst (e.g. Price et al. 2003; Sato et al. 2003; Uemura et al. 2003; Urata et al. 2004).

The optical afterglow was polarized at the $0.3-2.5 \%$ level, and the polarization was variable on time scales down to hours (Greiner et al. 2003b). The radio afterglow was not significantly polarized (Finkelstein et al. 2004; Taylor et al. 2005).

The optical afterglow decay can broadly be described using a broken power law with a break $\sim 3-8$ days after the burst (Lipkin et al. 2004). However, extensive observations of the optical afterglow light curve found a complex evolution of bumps and wiggles superimposed on the overall fading (e.g. Greiner et al. 2003b; Smith et al. 2003; Matheson et al. 2003; Bloom et al. 2004; Lipkin et al. 2004). These could have been due to structures in the forward and/or reverse shocks, or repeated energy injection from the central engine, or inhomogeneities in the ambient medium that the fireball expanded into (e.g. Sari \& Mészáros 2000; Berger et al. 2000; Granot et al. 2003; Piran et al. 2004). The interpretation of the various features in the light curve is therefore complicated.

The radio and millimeter afterglows were the brightest recorded for any burst to date (Berger et al. 2003b; Sheth et al. 2003; Kuno et al. 2004; Taylor et al. 2004; Kohno et al. 2005). The radio and millimeter light curves were initially approximately flat or slowly rising. After this, they broke to a power law $F_{v} \propto t^{-\delta}$ with a steep decay of $\delta \sim 2$. This is consistent with a jet break (e.g. Sari et al. 1999). The light curves for the observations from 43 to $250 \mathrm{GHz}$ all broke at $\sim 8$ days after the burst. In the single broken power law model, this would suggest that the jet break took place at $\sim 8$ days after the burst rather than at $\sim 3$ days which is allowed from the optical observations. The peak occurred at increasingly later times for longer wavelengths, for example, it was at $\sim 30$ days at $4.86 \mathrm{GHz}$. These longer wavelengths were below the synchrotron selfabsorption frequency $\left(v_{\mathrm{a}}\right)$ and/or below the typical synchrotron frequency for the lowest energy electron in the power law $\left(v_{\mathrm{m}}\right)$. Interstellar scintillation was an increasingly important factor below $15 \mathrm{GHz}$ for the early observations when the source was compact. At late times, the radio light curve showed an achromatic flattening which is consistent with the blast wave becoming transrelativistic (Frail et al. 2005).

A more complex model for the multiwavelength emission used breaks in the early optical afterglow decay at $\sim 0.25$ and $\sim 0.5$ days (e.g. Sato et al. 2003; Burenin et al. 2003). The first break could be due to the cooling break frequency $\left(v_{\mathrm{c}}\right)$ passing through the optical band. The second break would be similar to the one seen in X-rays; since it is achromatic, this suggests it is a jet break. However, an additional source of optical emission would then be required $\sim 1$ day after the burst. A possible model for the multiwavelength afterglow then uses two jets with different opening angles (Berger et al. 2003b; Sheth et al. 2003; Lipkin et al. 2004; Tiengo et al. 2004). The initial part of the optical and X-ray afterglow would come from a narrow ultra-relativistic jet. The late X-ray afterglow and optical brightening would come from a wide, mildly relativistic jet, with a jet break $\sim 10$ days after the burst. The plateau seen at longer wavelengths before the break could then be a convolution of the falling flux density (as $t^{-1 / 3}$ ) from the narrow angle jet and a rising flux density (as $t^{1 / 2}$ ) from the wide angle jet. Although this model describes the overall trends in the light curves, the multiple bumps and wiggles in the optical light curve still remain to be explained (Piran et al. 2004).

Beginning $~ 6$ days after the burst it became evident that there was an underlying component from the type Ic supernova SN 2003dh. The broad spectral features indicated a large expansion velocity similar to the type Ic hypernovae SN 1998bw and SN 1997ef (Stanek et al. 2003; Hjorth et al. 2003; Kawabata et al. 2003; Matheson et al. 2003; Mazzali et al. 2003; Kosugi et al. 2004). This was direct spectroscopic evidence that at least some classical GRBs originate from corecollapse supernovae. The SN 2003dh observations also indicated that the explosion was aspherical.

The host is believed to be a low to moderate metallicity starburst dwarf galaxy with $V \sim 22.7$ and $R>22.5$ (Fruchter et al. 2003; Matheson et al. 2003).

\section{SCUBA observing details}

SCUBA is the sub-millimeter continuum instrument for the JCMT (for a review see Holland et al. 1999). The observing, calibration, and reduction techniques used for GRB 030329 were the same as has been used on other bursts and are described in detail in Smith et al. (1999, 2001, 2005).

SCUBA uses two arrays of bolometers to simultaneously observe the same region of sky. The arrays are optimized for operations at 450 and $850 \mu \mathrm{m}$. Photometry observations are made using a single pixel of the arrays. The other bolometers in the arrays are used to perform a good sky noise subtraction (Archibald et al. 2002). During an observation, the secondary is chopped between the source and sky. The term "integration time" always refers to the "on+off" time, including the amount of time spent off-source. An $18 \mathrm{~s}$ integration thus amounts to a $9 \mathrm{~s}$ on-source observation time. A typical measurement consists of 50 integrations of $18 \mathrm{~s}$; we refer to this as a "run". Each observation of a source in general consists of several such runs, with focus, pointing, and calibration observations in between.

Version 1.6 of SURF ${ }^{1}$ (Jenness \& Lightfoot 2000) was used to combine the integrations, remove anomalous spikes, flatfield the array, and subtract the signal from the sky bolometers. The zenith sky opacity was determined using "skydips" in which the sky brightness temperature was measured at a range of elevations. This was used to apply atmospheric extinction corrections to the observed target fluxes. At least one standard

\footnotetext{
${ }^{1}$ SURF is distributed by the Starlink Project.
} 
Table 1. SCUBA $850 \mu \mathrm{m}(350 \mathrm{GHz})$ afterglow observations of GRB 030329.

\begin{tabular}{|c|c|c|c|c|c|c|}
\hline \multirow[t]{2}{*}{ Burst } & \multicolumn{2}{|c|}{ SCUBA observing times } & \multirow{2}{*}{$\begin{array}{l}\text { Time since } \\
\text { burst (days) }\end{array}$} & \multirow{2}{*}{$\begin{array}{l}\text { Integration } \\
\text { time }(\mathrm{sec})\end{array}$} & \multirow[t]{2}{*}{$\tau_{850}$} & \multirow{2}{*}{$\begin{array}{l}\text { Afterglow } 850 \mu \mathrm{m} \\
\text { flux density }(\mathrm{mJy})\end{array}$} \\
\hline & Start & Stop & & & & \\
\hline \multirow[t]{6}{*}{ GRB 030329} & 20030403.383 & 20030403.414 & 4.914 & 1800 & 0.623 & $37.8 \pm 4.5$ \\
\hline & 20030404.369 & 20030404.401 & 5.901 & 1800 & 0.467 & $27.6 \pm 2.9$ \\
\hline & 20030405.269 & 20030405.302 & 6.801 & 1800 & 0.356 & $33.9 \pm 2.8^{a}$ \\
\hline & 20030409.222 & 20030409.266 & 10.760 & 1836 & 0.433 & $10.9 \pm 5.1$ \\
\hline & 20030415.223 & 20030415.254 & 16.754 & 1800 & 0.300 & $-0.2 \pm 1.9$ \\
\hline & 20030416.339 & 20030416.370 & 17.870 & 1800 & 0.192 & $-1.9 \pm 2.7$ \\
\hline
\end{tabular}

${ }^{a}$ Not corrected for small pointing error.

calibration target was observed each night to determine the absolute flux of the GRB.

A typical integration time of $2 \mathrm{~h}$ gives an $\mathrm{rms} \sim 1.5 \mathrm{mJy}$ at $850 \mu \mathrm{m}$. However, the sensitivity depends significantly on the weather and the elevation of the source; since our ToO observations are done on short notice, sometimes these factors are less than ideal. Based on observed variations of the gain factor and signal levels we estimate typical systematic uncertainties in the absolute flux calibrations of $10 \%$ at $850 \mu \mathrm{m}$. In general the rms errors of the observations presented here are larger than this uncertainty.

The pointing of the JCMT is checked several times during the night to ensure that it is reliable. The pointing accuracy is usually a few arcsec. However, an error in the track model used between 2000 August 25 and 2003 April 25 resulted in pointing errors that were non-negligible (shifts larger than $4^{\prime \prime}$ ) for targets with elevations above $60^{\circ}$ and over small ranges of azimuth. Since the $850 \mu \mathrm{m}$ bolometric pixel has a diffraction limited resolution of $14^{\prime \prime}$, the target remains well within the bolometric pixel. However, there will be an error in the flux that is measured. For GRB 030329, the only problem was with a pointing calibration observation of 3C 273 on 2003 April 5. However, this did not significantly affect the result for that day.

\section{SCUBA observations of GRB 030329}

Unfortunately, SCUBA was not on the telescope at the time of the burst and it was several days before the first SCUBA observation could be made. The weather was not good for most of the observations leading to larger rms uncertainties than normal. Despite these difficulties, GRB 030329 was still easily detected at $850 \mu \mathrm{m}$ in the initial observations.

Table 1 summarizes all the $850 \mu \mathrm{m}$ SCUBA ToO observations for GRB 030329. These expand on and supersede the results in Hoge et al. (2003). The start times are the times when the GRB was first observed on each day, and the stop times are when the last observation of the GRB was completed. The time since the burst uses the mid-point between these start and stop times (although it should be noted that focus, pointing, and calibration observations may have also taken place in between the start and stop times); the time is the elapsed time in the Earth frame, not the rest frame of the host galaxy. The "integration time" for each observation is the "on+off" time; only half of this is spent on-source. The zenith optical depth at $850 \mu \mathrm{m}\left(\tau_{850}\right)$ is given for the time of the observation.
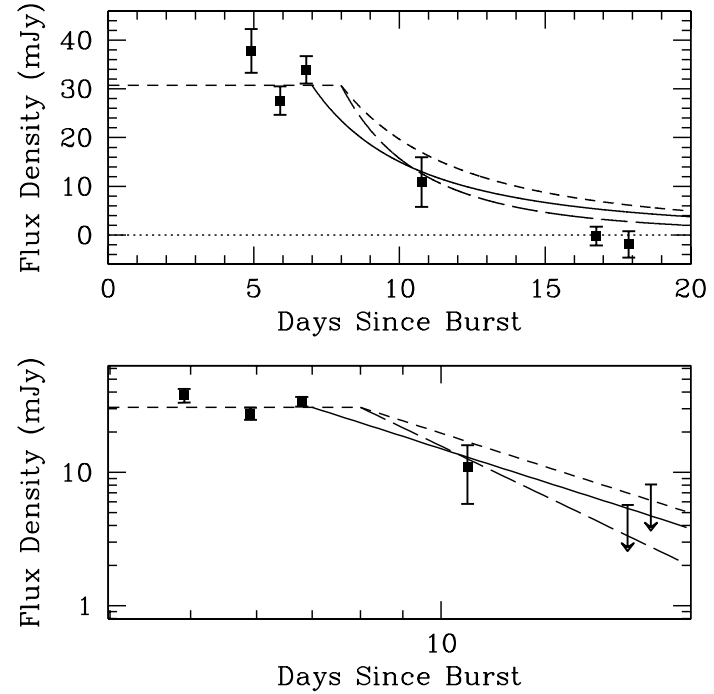

Fig. 1. The $850 \mu \mathrm{m}$ light curve for GRB 030329. The same data and models are plotted in the linear-linear (top) and log-log (bottom) versions, except that the last two data points are plotted as $3 \sigma$ upper limits in the latter. The initial dashed curve is a constant with $F_{v}=30.7 \mathrm{mJy}$. This breaks to $F_{v} \propto t^{-\delta}$, with the break at 8 days and $\delta=2$ (short dashed), the break at 8 days and $\delta=3$ (long dashed), and the break at 7 days and $\delta=2$ (solid).

The $30 \mathrm{mJy}$ source initially detected for GRB 030329 was by far the brightest SCUBA afterglow seen to date. For all the previous ToO observations performed by SCUBA, the $850 \mu \mathrm{m}$ flux density from the afterglow and/or reverse shock was $<10 \mathrm{mJy}$.

\subsection{SCUBA $850 \mu \mathrm{m}$ light curve}

The $850 \mu \mathrm{m}$ light curve is shown in Fig. 1. This is similar to the observations at longer wavelengths. The flux density is approximately constant initially, before breaking with a rapid decay. There is no evidence for an underlying dusty host galaxy.

The weighted mean for the first three SCUBA observations was $30.7 \pm 1.8 \mathrm{mJy}$ at $850 \mu \mathrm{m}$. This gives the constant dashed curve shown in Fig. 1 for the first week.

Unfortunately, we do not have high quality observations during the decay phase, due to the terrible weather at that time. However, the lack of detection 17 days after the burst allows us to use simple qualitative models to constrain the break time and/or the decay slope. 
The short dashed curve in Fig. 1 shows a break at 8 days after the burst and a decay of $\delta=2$. This would predict a flux density of $7.0 \mathrm{mJy}$ at 16.8 days after the burst. This is therefore inconsistent with our non-detection at the $3.7 \sigma$ level.

To maintain $\delta=2$ for the whole decay, it would be necessary for the break to occur earlier. The solid curve in Fig. 1 places the break at 7 days. This is just consistent with our $3 \sigma$ limit at 16.8 days.

To have the jet break occur at later times, it would be necessary to have a faster decay. For example, the long dashed curve in Fig. 1 uses a break at 8 days and $\delta=3$. Such a steep decay (on average) has not been seen in the other longer wavelength observations of this burst. However, as shown in Sect. 4.2, it is possible that a break moved through the sub-millimeter band between 7 and 17 days after the burst, and this might account for a faster decay. Alternatively, Kohno et al. (2005) found that the flux density at $93 \mathrm{GHz}$ was approximately constant from 12 to 17 days after the burst before a rapid drop took place between 17 and 18 days after the burst. It is therefore possible that a similar rapid drop took place $\lesssim 16$ days after the burst at $850 \mu \mathrm{m}$.

The SCUBA flux density at $850 \mu \mathrm{m}$ before the break in the light curve is consistent with being constant. Sheth et al. (2003) found a small $(<20 \%)$ drop in the flux density at $100 \mathrm{GHz}$ starting April 2 followed by a rise to its mean level on April 5. This is similar to our observation on April 4 being lower than on April 3 and 5. On April 5, the flux density at $43.3 \mathrm{GHz}$ was the highest recorded, and the flux density at the four other longer wavelengths measured was also temporarily higher on that day (Berger et al. 2003b). Thus this variability may have been real. If so, it complicates the determination of the actual time for the break.

The observations during each SCUBA run show no significant variability. One possible explanation for the fluctuations in the optical light curve is that the central engine had multiple ejections, rather than a single episode (e.g. Granot et al. 2003). This would lead to refreshed shocks when the faster moving ejecta caught up with slower moving material. A prediction of this model is that there should be short-lived reverse shocks when the ejecta collide producing significant longer wavelength flares. The lack of large-scale variability at $850 \mu \mathrm{m}$ agrees with the results from other observations (Sheth et al. 2003), and argues against this model. However, we caution that our data only sparsely sample the light curve. Unfortunately, we do not have any SCUBA data during the first few days to look for a bright flash that might be expected in the two component jet model (Piran et al. 2004).

\subsection{Evolution of the longer wavelength spectrum}

Due to the poor observing conditions, the source was not detected at $450 \mu \mathrm{m}$ in any of the individual observations. The source was also not detected at $450 \mu \mathrm{m}$ when the results from separate days are combined. The weighted mean for the first three days was $-69 \pm 33 \mathrm{mJy}$ at $450 \mu \mathrm{m}$.

Figure 2 illustrates the longer wavelength spectrum before the break in the light curve using the average fluxes for

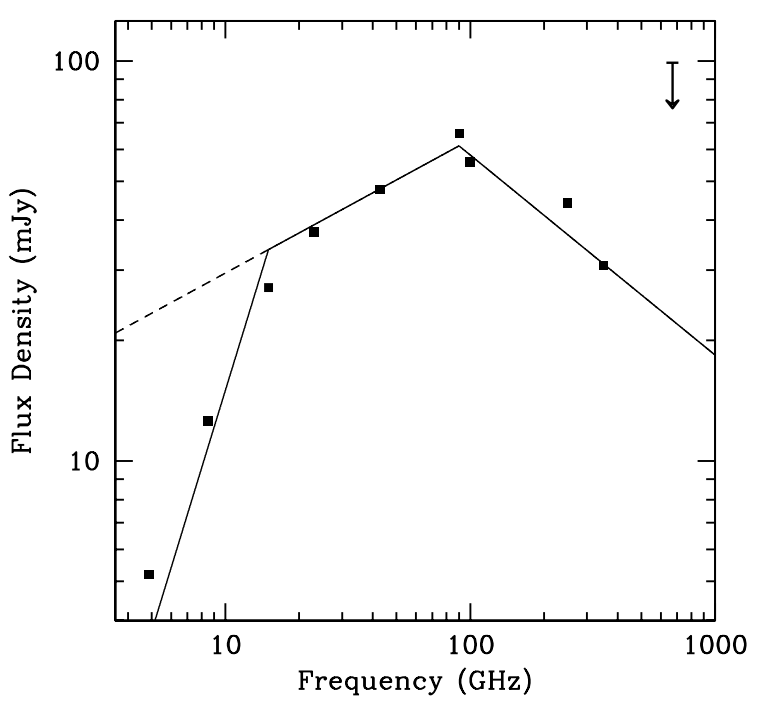

Fig. 2. The longer wavelength spectrum for GRB 030329 before the break in the light curve. The data points show typical averaged values for $\sim 6$ days after the burst. Added to the $850 \mu \mathrm{m}(350 \mathrm{GHz})$ and $450 \mu \mathrm{m}(670 \mathrm{GHz})$ data from this paper are the results at 4.86, 8.46 and $15 \mathrm{GHz}$ (Berger et al. 2003b), 23 and $43 \mathrm{GHz}$ (Berger et al. 2003b; Kuno et al. 2004), $90 \mathrm{GHz}$ (Kuno et al. 2004), 100 and $250 \mathrm{GHz}$ (Sheth et al. 2003). The upper limit at $450 \mu \mathrm{m}$ is $3 \sigma$. The solid curve is $F_{v}=0.15 v^{2}$ up to $15 \mathrm{GHz}, F_{v}=13.7 v^{1 / 3}$ up to a peak of $61 \mathrm{mJy}$ at $90 \mathrm{GHz}$, and $F_{v}=582 v^{-1 / 2}$ after the peak.

the observations $\sim 6$ days after the burst. Since data has been combined from different observatories, and the source may be variable, the points will have uncertainties of up to $\sim 20 \%$. However, this does give a good qualitative picture for the longer wavelength spectrum at the time before the break.

The solid curve in Fig. 2 shows one qualitative fit to the data. The rise uses $F_{v} \propto v^{2}$ up to $15 \mathrm{GHz}$ and $F_{v} \propto v^{1 / 3}$ up to a peak of $61 \mathrm{mJy}$ at $90 \mathrm{GHz}$. The fall is $F_{v} \propto v^{-1 / 2}$. The short dashed curve extrapolates the $v^{1 / 3}$ curve to highlight the break at longer wavelengths.

Spectral indices such as these are commonly found in synchrotron fireball models (e.g. Sari et al. 1998; Piran 1999; Wijers \& Galama 1999; Chevalier \& Li 2000; Granot et al. 2000; Granot \& Sari 2002; Panaitescu \& Kumar 2004). For example, the breaks could be $v_{\mathrm{a}}=15 \mathrm{GHz}$ and $v_{\mathrm{m}}=90 \mathrm{GHz}$.

For the $R$ band $\left(4.2 \times 10^{5} \mathrm{GHz}\right)$ this fit would extrapolate to give $0.9 \mathrm{mJy}$ or $R \sim 16$. The source was that bright $\sim 1$ day after the burst, and was at $R \sim 18$ at 6 days after the burst. In this case, it would then be necessary to have another break in the spectrum between the sub-millimeter and optical bands. For example, this could be the cooling break $v_{\mathrm{c}}$.

Figure 3 illustrates the longer wavelength spectrum after the break in the light curve using the average fluxes for the observations $\sim 17$ days after the burst. Again, the uncertainties in the points may be up to $\sim 20 \%$. The solid curve in Fig. 3 shows one qualitative fit to the data, with $F_{v} \propto v^{2}$ up to $8 \mathrm{GHz}$, $F_{v} \propto v^{1 / 3}$ up to a peak of $27 \mathrm{mJy}$ at $24 \mathrm{GHz}$, and a fall with $F_{v} \propto v^{-1 / 2}$

The short dashed curve in Fig. 3 shows the fit from Fig. 2. Qualitatively, the spectral indices have stayed the same, with the two longer wavelength breaks $\left(v_{\mathrm{a}}\right.$ and $v_{\mathrm{m}}$ in the example 


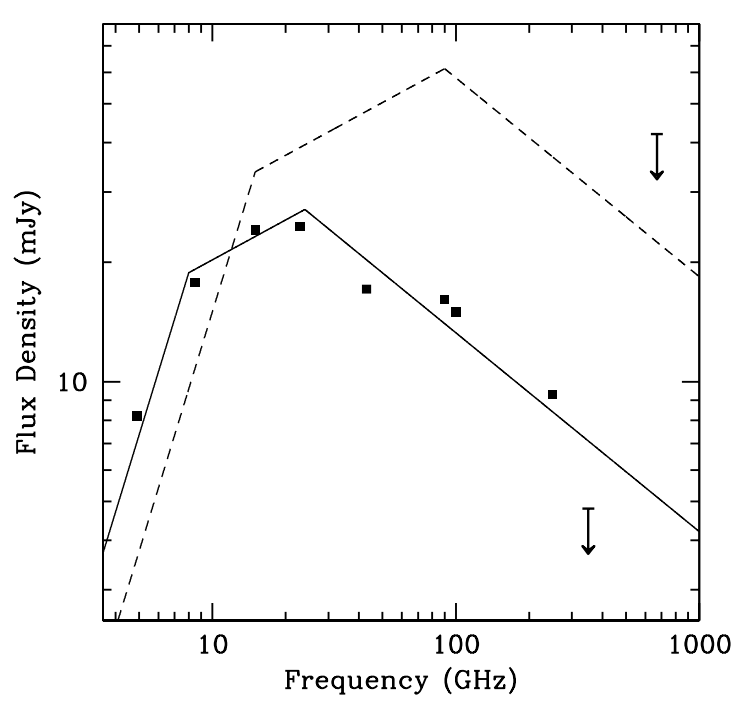

Fig. 3. The longer wavelength spectrum for GRB 030329 after the break in the light curve. The data points show typical averaged values for $\sim 17$ days after the burst. Added to the $3 \sigma 850 \mu \mathrm{m}(350 \mathrm{GHz})$ and $450 \mu \mathrm{m}(670 \mathrm{GHz})$ limits from this paper are the results at 4.86, 8.46, 15, and $43 \mathrm{GHz}$ (Berger et al. 2003b), $23 \mathrm{GHz}$ (Kuno et al. 2004), $93 \mathrm{GHz}$ (Kohno et al. 2005), 100 and $250 \mathrm{GHz}$ (Sheth et al. 2003). The solid curve is $F_{v}=0.294 v^{2}$ up to $8 \mathrm{GHz}, F_{v}=9.41 v^{1 / 3}$ up to a peak of $27 \mathrm{mJy}$ at $24 \mathrm{GHz}$, and $F_{v}=133 v^{-1 / 2}$ after the peak. The short dashed curve shows the fit from Fig. 2.

above) moving to longer wavelengths and the flux at the peak falling.

However, the model predicts a flux density of $7.1 \mathrm{mJy}$ at $850 \mu \mathrm{m}$ at $\sim 17$ days after the burst. This is inconsistent with our lack of detection at the $3.7 \sigma$ level if we use the limit from April 15, and at the $4.4 \sigma$ level if we combine our last two SCUBA observations.

One way to reduce the disagreement between the model and the SCUBA observation at $\sim 17$ days after the burst is if the third break ( $v_{\mathrm{c}}$ in the example above) passed through the sub-millimeter band shortly before our last observations. This could also help to explain why the decay of the $850 \mu \mathrm{m}$ light curve was faster than $\delta=2$. However, the spectral break would need to be sharp to fully explain the SCUBA limit. Also, in the simple fireball models, $v_{\mathrm{c}}$ should remain constant after the jet break. Thus this would require a more complex model, such as having the magnetic energy change with time (e.g. Yost et al. 2003).

An alternative explanation for the lack of detection at $850 \mu \mathrm{m}$ at $\sim 17$ days after the burst is that there could have been a rapid drop in the light curve, as discussed in Sect. 4.1. The drop must have taken place earlier than 16.8 days after the burst at $850 \mu \mathrm{m}$. Figure 3 shows that the $250 \mathrm{GHz}$ point was still on the $v^{-1 / 2}$ curve at 16.3 days after the burst. The drop took place between 17 and 18 days after the burst at $93 \mathrm{GHz}$. This indicates that if the drop is real, it did not occur at exactly the same time for all wavelengths. Instead, it swept from shorter to longer wavelengths.
Table 2. Host galaxy fluxes for GRB 030329 determined by combining the SCUBA $850 \mu \mathrm{m}$ and $450 \mu \mathrm{m}$ observations taken on the last two days.

\begin{tabular}{llll}
\hline \hline Burst & Redshift & \multicolumn{2}{c}{ Host flux density (mJy) } \\
& & $850 \mu \mathrm{m}$ & $450 \mu \mathrm{m}$ \\
\hline GRB 030329 & 0.1685 & $-0.8 \pm 1.6$ & $17 \pm 14$ \\
\hline
\end{tabular}

\subsection{SCUBA host galaxy limits}

The results in Table 2 show the weighted means for the last two days of SCUBA observations. Although a small amount of afterglow emission may be present, this contamination should be small. There is no indication for any sub-millimeter emission from the host galaxy.

It is not surprising that GRB 030329 is not a bright quiescent sub-millimeter source given that (1) it is at a low redshift; (2) the host is probably a dwarf galaxy; and (3) the X-ray and optical observations show relatively little excess absorption from dust above the Galactic value (Tiengo et al. 2003; Matheson et al. 2003). However, given the short observing times and less than ideal observing conditions, the limits on the host galaxy fluxes are less restrictive than for some of the other bursts whose host galaxies have been observed by SCUBA (Barnard et al. 2003; Berger et al. 2003a; Tanvir et al. 2004; Smith et al. 2005).

The SCUBA host galaxy compilation of Tanvir et al. (2004) focused on hosts with $850 \mu \mathrm{m}$ rms flux densities $<1.4 \mathrm{mJy}$. Since our rms is slightly higher than this, and the redshift of GRB 030329 is $<2$, the lack of detection of a sub-millimeter host galaxy does not shed any new light on the question of whether GRBs are closely linked to the most luminous dusty star-forming galaxies.

\section{Summary}

GRB 030329 was by far the brightest sub-millimeter afterglow seen to date. Despite our limited observations, we were still able to constrain the break and/or decay light curve. Assuming that the decay is not steeper than $\delta=2$, the break must have taken place $\sim 7$ days after the burst at $850 \mu \mathrm{m}$. This jet break could have been at a slightly later time if another break (perhaps the cooling break) passed through the sub-millimeter band between 7 and 17 days after the burst, or if there was a drop in the $850 \mu \mathrm{m}$ flux at $\lesssim 16$ days after the burst.

No short-lived large-scale brightenings were detected in the sub-millimeter light curve. The flux density at $850 \mu \mathrm{m}$ was consistent with being constant up to the jet break. However, the $850 \mu \mathrm{m}$ results also agree with those at longer wavelengths that show a slightly brighter flux $\sim 7$ days after the burst, right at the time of the break.

The peak of the afterglow emission was at $\sim 90 \mathrm{GHz}$ in the days before the break in the light curve. The spectral indices were qualitatively given by +2 and $+1 / 3$ up to the peak and $-1 / 2$ beyond it. A simple modeling is consistent with the spectral indices remaining the same as the afterglow evolved, with the spectral breaks moving to longer wavelengths at later times and the flux at the peak falling. 
GRB 030329 did not have any significant sub-millimeter contribution from the host galaxy.

The observations of GRB 030329 show that for bright afterglows it is important to monitor the flux evolution carefully to look for fluctuations in the light curve and to determine the breaks. Observations of new bursts are continuing to produce surprises, and there is much left to learn about GRB afterglows and host galaxies. To obtain a complete picture of their nature will require the careful study of many bursts to expand our sample. Sub-millimeter observations with a $\sim$ mJy sensitivity are a key component to the multi-wavelength coverage. To this end, our program of ToO observations using SCUBA is ongoing.

Acknowledgements. The James Clerk Maxwell Telescope is operated by The Joint Astronomy Centre on behalf of the Particle Physics and Astronomy Research Council of the UK, The Netherlands Organisation for Scientific Research, and the National Research Council of Canada.

We thank the JCMT Director Gary Davis for authorizing the ToO observations. We are indebted to all the observers whose time was displaced by these observations, and acknowledge the dedicated efforts of the JCMT telescope operators Jim Hoge and Jonathan Kemp for their valuable assistance with the observations. We thank the referee for a prompt review and helpful suggestions.

We are grateful to Scott Barthelmy and Paul Butterworth for maintaining the GRB Coordinates Network (GCN), and to the other groundbased observers for the rapid dissemination of their burst results.

We acknowledge the data analysis facilities provided by the Starlink Project which is run by CCLRC on behalf of PPARC.

The work at Rice University was supported in part by AFOSR/NSF grant number NSF AST-0123487.

\section{References}

Archibald, E. N., Jenness, T., Holland, W. S., et al. 2002, MNRAS, 336,1

Barnard, V. E., Blain, A. W., Tanvir, N. R., et al. 2003, MNRAS, 338,1

Berger, E., Sari, R., \& Frail, D. A. 2000, ApJ, 545, 56

Berger, E., Cowie, L. L., Kulkarni, S. R., et al. 2003a, ApJ, 588, 99

Berger, E., Kulkarni, S. R., Pooley, G., et al. 2003b, Nature, 426, 154

Bloom, J. S., Frail, D. A., Kulkarni, S. R., et al. 1998, ApJ, 508, L21

Bloom, J. S., van Dokkum, P. G., Bailyn, C. D., et al. 2004, ApJ, 127, 252

Burenin, R. A., Sunyaev, R. A., Pavlinksy, M. N., et al. 2003, Astron. Lett., 29, 573

Chevalier, R. A., \& Li, Z.-Y. 2000, ApJ, 536, 195

Finkelstein, A. M., Ipatov, A. V., Gnedin, Y. N., et al. 2004, Astron. Lett., 30,368

Frail, D. A., Berger, E., Galama, T., et al. 2000, ApJ, 538, L129

Frail, D. A., Bertoldi, F., Moriarty-Schieven, G. H., et al. 2002, ApJ, 565,829

Frail, D. A., Yost, S. A., Berger, E., et al. 2003, ApJ, 590, 992

Frail, D. A., Soderberg, A. M., Kulkarni, S. R., et al. 2005, ApJ, 619, 994

Fruchter, A., Levan, A., Hook, R., et al. 2003, GCN, 2243

Galama, T. J., Briggs, M. S., Wijers, R. A. M. J., et al. 1999, Nature, 398,394

Granot, J., \& Sari, R. 2002, ApJ, 568, 820

Granot, J., Piran, T., \& Sari, R. 2000, ApJ, 534, L163

Granot, J., Nakar, E., \& Piran, T. 2003, Nature, 426, 138

Greiner, J., Peimbert, M., Estaban, C., et al. 2003a, GCN, 2020
Greiner, J., Klose, S., Reinsch, K., et al. 2003b, Nature, 426, 157

Hjorth, J., Sollerman, J., Møller, P., et al. 2003, Nature, 423, 847

Hoge, J. C., Meijerink, R., Tilanus, R. P. J., \& Smith, I. A. 2003, GCN, 2088

Holland, W. S., Robson, E. I., Gear, W. K., et al. 1999, MNRAS, 303, 659

Jenness, T., \& Lightfoot, J. F. 2000, Starlink User Note 216, Starlink Project, CLRC

Kawabata, K. S., Deng, J., Wang, L., et al. 2003, ApJ, 593, L19

Kohno, K., Tosaki, T., Okuda, T., et al. 2005, PASJ, 57, 147

Kosugi, G., Mizumoto, Y., Kawai, N., et al. 2004, PASJ, 56, 61

Kulkarni, S. R., Frail, D. A., Sari, R., et al. 1999, ApJ, 522, L97

Kuno, N., Sato, N., Nakanishi, H., et al. 2004, PASJ, 56, L1

Lipkin, Y. M., Ofek, E. O., Gal-Yam, A., et al. 2004, ApJ, 606, 381

Marshall, F. E., \& Swank, J. H. 2003, GCN, 1996

Marshall, F. E., Markwardt, C., \& Swank, J. H. 2003, GCN, 2052

Matheson, T., Garnavich, P. M., Stanek, K. Z., et al. 2003, ApJ, 599, 394

Mazzali, P. A., Deng, J., Tominaga, N., et al. 2003, ApJ, 599, L95

Mészáros, P. 2002, ARA\&A, 40, 137

Panaitescu, A., \& Kumar, P. 2004, MNRAS, 350, 213

Piran, T. 1999, Phys. Rep., 314, 575

Piran, T., Nakar, E., \& Granot, J. 2004, in Gamma-Ray Bursts: 30 Years of Discovery: Gamma-Ray Burst Symposium, ed. E. E. Fenimore, \& M. Galassi, AIP Conf. Proc. (NY: AIP), 727, 181

Price, P. A., Fox, D. W., Kulkarni, S. R., et al. 2003, Nature, 423, 844 Sari, R., \& Mészáros, P. 2000, ApJ, 535, L33

Sari, R., Piran, T., \& Narayan, R. 1998, ApJ, 497, L17

Sari, R., Piran, T., \& Halpern, J. P. 1999, ApJ, 519, L17

Sato, R., Kawai, N., Suzuki, M., et al. 2003, ApJ, 599, L9

Schnoor, P. W., Welch, D. L., Fishman, G. J., \& Price, A. 2003, GCN, 2176

Sheth, K., Frail, D. A., White, S., et al. 2003, ApJ, 595, L33

Smith, D. A., Rykoff, E. S., Akerlof, C. W., et al. 2003, ApJ, 596, L151

Smith, I. A., Tilanus, R. P. J., Van Paradijs, J., et al. 1999, A\&A, 347, 92

Smith, I. A., Van Paradijs, J., Tilanus, R. P. J., et al. 2000, in Gamma-Ray Bursts: 5th Huntsville Symposium, ed. R. M. Kippen, R. S. Mallozzi, \& G. J. Fishman (New York: AIP), 326

Smith, I. A., Tilanus, R. P. J., Wijers, R. A. M. J., et al. 2001, A\&A, 380,81

Smith, I. A., Tilanus, R. P. J., Tanvir, N., et al. 2005, A\&A, 439, 987

Stanek, K. Z., Matheson, T., Garnavich, P. M., et al. 2003, ApJ, 591, L17

Tanvir, N. R., Barnard, V. E., Blain, A. W., et al. 2004, MNRAS, 352 , 1073

Taylor, G. B., Frail, D. A., Berger, E., \& Kulkarni, S. R. 2004, ApJ, 609, L1

Taylor, G. B., Momjian, E., Pihlstrom, Y., Ghosh, T., \& Salter, C. 2005, ApJ, 622, 986

Tiengo, A., Mereghetti, S., Ghisellini, G., et al. 2003, A\&A, 409, 983

Tiengo, A., Mereghetti, S., Ghisellini, G., et al. 2004, A\&A, 423, 861

Torii, K., Kato, T., Yamaoka, H., et al. 2003, ApJ, 597, L101

Uemura, M., Kato, T., Ishioka, R., et al. 2003, Nature, 423, 843

Urata, Y., Miyata, T., Nishiura, S., et al. 2004, ApJ, 601, L17

Vanderspek, R., Sakamoto, T., Barraud, C., et al. 2004, ApJ, 617, 1251

Van Paradijs, J., Kouveliotou, C., \& Wijers, R. A. M. J. 2000, ARA\&A, 38, 379

Wijers, R. A. M. J., \& Galama, T. J. 1999, ApJ, 523, 177

Yost, S. A., Frail, D. A., Harrison, F. A., et al. 2002, ApJ, 577, 155

Yost, S. A., Harrison, F. A., Sari, R., \& Frail, D. A. 2003, ApJ, 597, 459 